MANCHESTER

1824

넌

Economics

Discussion Paper Series

EDP-1108

\title{
Existence, Incentive Compatibility and Efficiency of the Rational Expectations Equilibrium
}

\author{
Yeneng Sun \\ Lei $\mathrm{Wu}$ \\ Nicholas C. Yannelis
}

January 2011

Economics

School of Social Sciences

The University of Manchester

Manchester M13 9PL 


\title{
Existence, Incentive Compatibility and Efficiency of the Rational Expectations Equilibrium
}

\author{
Yeneng Sun*, Lei $\mathrm{Wu}^{\dagger}$ and Nicholas C. Yannelis ${ }^{\ddagger}$
}

\begin{abstract}
The rational expectations equilibrium (REE), as introduced in Radner (1979) in a general equilibrium setting $\grave{a}$ la Arrow-Debreu-Mckenzie, often fails to have normative properties such as universal existence, incentive compatibility and efficiency. We resolve those problems by providing a new model which makes the standard REE a desirable solution concept. In particular, we consider an asymmetric information economy with a continuum of agents whose private signals are independent conditioned on the macro states of nature; for such an economy, the REE universally exists, is incentive compatible and efficient. Also, we introduce the notion of REE with aggregate signals which extends the standard REE concept by allowing agents to use the information generated by a macroeconomic statistic, namely agents' aggregate signals. It is shown that this new REE concept also possesses the desirable properties as the standard REE does, but with much more general conditions on agents' utility functions.
\end{abstract}

*Department of Economics, National University of Singapore, 1 Arts Link, Singapore 117570. e-mail: ynsun@nus.edu.sg

$\dagger$ Department of Mathematics, National University of Singapore, 2 Science Drive 2, Singapore 117543. e-mail: lwu@nus.edu.sg

${ }^{\ddagger}$ Department of Economics, University of Illinois at Urbana-Champaign, Champaign, IL 61820 . e-mail: nyanneli@uiuc.edu; and Economics - School of Social Sciences, The University of Manchester, Oxford Road, Manchester M13 9PL, UK. e-mail: nicholasyannelis@manchester.ac.uk 


\section{Introduction}

In seminal papers, Radner (1979) and Allen (1981) extended the finite agent ArrowDebreu-McKenzie economy to allow for asymmetric information. In this asymmetric information economy, each agent is characterized by a random utility function, random initial endowment, private information with a prior. The equilibrium notion that Radner put forward is called rational expectations equilibrium (REE), which is an extension of the deterministic Walrasian equilibrium of the Arrow-Debreu-McKenzie model. According to the REE, each individual maximizes interim expected utility conditioned on her own private information as well as the information generated by the equilibrium price.

By now it is well known that in a finite agent economy with asymmetric information, a rational expectations equilibrium, may not exist $^{1}$ (see Kreps (1977)), may not be incentive compatible, may not be Pareto optimal and may not be implementable as a perfect Bayesian equilibrium of an extensive form game (see Glycopantis et al. (2005), p. 31 and also Example 9.1.1, p. 43). Thus, if the intent of the REE notion is to capture contracts among agents under asymmetric information, then such contacts not only do they not exist universally in well behaved economies (i.e., economies with concave, continuous, monotone utility functions and strictly positive initial endowments), but even if they exist, they fail to have any normative properties, such as incentive compatibility, Pareto optimality and Bayesian rationality.

The main difficulty that one encounters with the REE in a finite agent economy is the fact that individuals are supposed to maximize their interim expected utility conditioned not only on their own private information, but also on the information generated by the equilibrium price (recall that the equilibrium price is endogenously determined); at the same time, the individuals can influence the equilibrium price to their own benefit by manipulating their private information. However, this would not have been a problem if each agent's private information is negligible. This poses the following question. Is it possible to model the REE in such a way that each agent's effect on the equilibrium price is negligible and therefore the REE concept overcomes the difficulties encountered above?

We introduce a new model where the REE concept becomes free of the problems mentioned above. In this model, agents' perceived private signals conditioned on an exogenously given macro state of nature are independent, ${ }^{2}$ and thus, by the exact law of large numbers in Sun (2006), the influence of each agent on the equilibrium price is negligible. In such a framework, the equilibrium price only reveals some information about the macro states. In particular, our Corollary 2 shows the existence of a REE, which is incentive compatible and ex-post Pareto optimal (as mentioned earlier, these

\footnotetext{
${ }^{1}$ It only exists in a generic sense as Radner (1979) and Allen (1981) have shown.

${ }^{2}$ It follows from Theorem 1 of Hammond and Sun (2008) that the measurability assumption imposed in this paper always implies the existence of such a conditional independence structure; see Subsection 2.4 for more discussion.
} 
results are false in a finite agent economy). Note that the REE price in this theorem depends on the exogenously given macro states of nature. Since an individual agent in such an economy cannot influence the macro states, the effect of each agent on the equilibrium price is exactly negligible, which assures incentive compatibility. The above result is based on the assumption that the utility of each agent depends only on her private information and is strictly concave. In contrast, Corollary 1 drops both conditions when we consider the special case that there is only one macro state (i.e, the private signals are unconditionally independent). In this case, the resulting REE price is constant.

Macroeconomic statistics can often provide a good description of an economy. In view of this, we introduce a macroeconomic statistic - aggregate signals into the REE model. In particular, each agent maximizes her expected utility conditioned on her private information and also on the information generated by the price and aggregate signals. The corresponding equilibrium concept is called REE with aggregate signals. The advantage for allowing the agents to use this additional information is that strict concavity and the dependence of the utility functions on the private information are no longer needed. What we need is just the mild assumption that agents' private signals are independent conditioned on the macro states of nature. Specifically, Theorem 1 shows the existence of an incentive compatible, interim efficient REE without strict concavity and without the dependence of the utility functions on the private information. This extension of the Radner-Allen REE model is different from previous work in the literature since none of these models take into account the information carried in the aggregate signals.

The REE and REE with aggregate signals are often equivalent. In particular, Lemma 1 below shows that they are equivalent when the equilibrium price fully reveals the macro states. Lemma 2 also shows their equivalence under strict concavity and dependence of the utility functions on the private information. Based on those equivalence results, we only need to give a complete proof for results on the REE with aggregate signals; results on the REE will follow as corollaries.

This paper is organized as follows. In Section 2, we present the economic model, the notions of REE, Pareto optimality and incentive compatibility, and some assumptions. The main result and its corollaries are stated in Section 3. In Section 4, we discuss related literature. Some concluding remarks are provided in Section 5. The proofs are given in Section 6.

\section{The Economic Model}

In this section, we define the notion of a private information economy, followed by the definitions of rational expectations equilibrium, rational expectations equilibrium with aggregate signals, Pareto optimality and incentive compatibility. 


\subsection{Private information economy}

We consider an atomless probability space ${ }^{3}(I, \mathcal{I}, \lambda)$ as the space of agents. Each agent receives a private signal of type $q \in T^{0}=\left\{q_{1}, q_{2}, \ldots, q_{L}\right\}^{4}$. Let $\mathcal{T}^{0}$ denote the power set of $T^{0}$. A signal profile $t$ is a function from $I$ to $T^{0}$. For $i \in I, t(i)$ (also denoted by $t_{i}$ ) is the private signal of agent $i$ while $t_{-i}$ is the restriction of $t$ to the set $I \backslash\{i\}$. Let $(T, \mathcal{T}, P)$ be a probability space that models the uncertainty associated with the private signal profiles for all the agents. ${ }^{5}$ For simplicity, we shall assume that $(T, \mathcal{T})$ has a product structure so that $T$ is the product of $T_{-i}$ and $T^{0}$, while $\mathcal{T}$ is the product $\sigma$-algebra of $\mathcal{T}^{0}$ and a $\sigma$-algebra $\mathcal{T}_{-i}$ on $T_{-i}$. For $t \in T$ and $t_{i}^{\prime} \in T^{0}$, we shall adopt the usual notation $\left(t_{-i}, t_{i}^{\prime}\right)$ to denote the signal profile whose value is $t_{i}^{\prime}$ for $i$ and $t_{j}$ for $j \neq i$.

The private signal process is a function from $I \times T$ to $T^{0}$ such that $f(i, t)=t_{i}$ for any $(i, t) \in I \times T$. For each $i \in I$, let $\tilde{t}_{i}$ be the projection mapping from $T$ to $T^{0}$ with $\tilde{t}_{i}(t)=t_{i}$. The private signal distribution $\tau_{i}$ of agent $i$ over $T^{0}$ is defined as $\tau_{i}(\{q\})=P\left(\tilde{t}_{i}=q\right)$ for $q \in T^{0} . P^{T_{-i}}(\cdot \mid q)$ is the conditional probability measure on the measurable space $\left(T_{-i}, \mathcal{T}_{-i}\right)$ when the private signal of agent $i$ is $q \in T^{0}$. If $\tau_{i}(\{q\})>0$, then it is clear that for $D \in \mathcal{T}_{-i}, P^{T_{-i}}(D \mid q)=P(D \times\{q\}) / \tau_{i}(\{q\})$.

We also would like to include another source of uncertainty in our model - the macro level uncertainty. Let $S=\left\{s_{1}, s_{2}, \ldots, s_{K}\right\}$ be the set of all possible macro states of nature, and $\mathcal{S}$ the power set of $S$. The $S$-valued random variable $\tilde{s}$ on $T$ models the macro level uncertainty. For each macro state $s \in S$, denote the event $(\tilde{s}=s)=\{t \in T: \tilde{s}(t)=s\}$ that $s$ occurs by $C_{s}$. The probability that $s$ occurs is $\pi_{s}=P\left(C_{s}\right)$. Without loss of generality, assume that $\pi_{s}>0$ for each $s \in S$. Let $P_{s}$ be the conditional probability measure on $(T, \mathcal{T})$ when the random variable $\tilde{s}$ takes value s. Thus, for each $B \in \mathcal{T}, P_{s}(B)=P\left(C_{s} \cap B\right) / \pi_{s}$. It is obvious that $P=\sum_{s \in S} \pi_{s} P_{s}$. Note that the conditional probability measure $P_{s}$ is often denoted as $P(\cdot \mid s)$ in the literature.

The common consumption set for all the agents is the positive orthant $\mathbb{R}_{+}^{m}$. Let $u$ be a function from $I \times \mathbb{R}_{+}^{m} \times T$ to $\mathbb{R}_{+}$such that for any given $i \in I, u(i, z, t)$ is the utility of agent $i$ at consumption bundle $z \in \mathbb{R}_{+}^{m}$ and signal profile $t \in T$. For any given $(i, t) \in I \times T$, we assume that $u(i, z, t)$ (also denoted by $u_{(i, t)}(z)$ or $\left.u_{i}(z, t)\right)^{6}$ is continuous, monotonic in $z \in \mathbb{R}_{+}^{m} \cdot{ }^{7}$ For each $z \in \mathbb{R}_{+}^{m}, u_{z}(\cdot, \cdot)$ is an integrable function

\footnotetext{
${ }^{3}$ We use the convention that all probability spaces are countably additive.

${ }^{4}$ In the literature, one usually assumes that different agents have different sets of private signals and requires that agents receive each of them with positive probability. For notational simplicity, we choose to work with a common set $T^{0}$ of private signals, but allow zero probability for some of the redundant signals. There is no loss of generality in this latter approach.

${ }^{5}$ Thus $T$ is a space of functions from $I$ to $T^{0}$.

${ }^{6}$ In the sequel, we shall often use subscripts to denote some variable of a function that is viewed as a parameter in a particular context.

${ }^{7}$ The utility function $u(i, \cdot, t)$ is monotonic if for any $y, z \in \mathbb{R}_{+}^{m}$ with $y \leq z$ and $y \neq z, u(i, y, t)<$ $u(i, z, t)$.
} 
on $I \times T .^{8}$

In our model, the initial endowment of an agent depends on her private signal. The initial endowment profile $e$ is a function from $I \times T^{0}$ to $\mathbb{R}_{+}^{m}$ such that for $(i, q) \in I \times T^{0}, e(i, q)$ is the initial endowment of agent $i$ when her private signal is $q$. We assume that for each $q \in T^{0}, e(\cdot, q)$ is $\lambda$-integrable over $I$, and $\int_{I} e(i, q) d \lambda$ is in the strictly positive cone $\mathbb{R}_{++}^{m}{ }^{9}$.

Formally, the private information economy is denoted by

$$
\mathcal{E}=\left\{I \times T, u, e, f,\left(\tilde{t}_{i}, i \in I\right), \tilde{s}\right\} .
$$

\subsection{Rational expectations equilibrium, incentive compatibility and effi- ciency}

In this subsection, we define the notion of a rational expectations equilibrium. Since the reports of agents' private signals play a key role for determining the equilibrium price, the issue of incentive compatibility also arises.

A price is a normalized nonnegative vector $p$ in $\Delta_{m}$, where $\Delta_{m}$ is the unit simplex of $\mathbb{R}_{+}^{m}$. A price process $\tilde{p}$ is a function from $T$ to $\Delta_{m}$. For each $t \in T, \tilde{p}(t)$ is the price when the signal profile is $t$. For notational simplicity, the letter $p$ will be used both for a price and a price process. The terms "price" and "price process" are used synonymously in this paper.

As we allow the agents to use their private information and the information revealed by the equilibrium price, we shall define an allocation $x$ to be a measurable mapping from $I \times \Delta_{m} \times T^{0}$ to $\mathbb{R}_{+}^{m}$. For each $(i, p, q) \in I \times \Delta_{m} \times T^{0}, x(i, p, q)$ is the consumption bundle of agent $i$ when the price is $p$ and her private signal is $q$.

Since an agent's initial endowment is contingent on her private signal $q$, we denote the budget set for agent $i$ by $B_{i}(p, q)$ when the price is $p$ and her private signal is $q$. Hence, $B_{i}(p, q)=\left\{z \in \mathbb{R}_{+}^{m}: p z \leq p e(i, q)\right\}$.

Given a consumption bundle $z \in \mathbb{R}_{+}^{m}$, a private signal $q \in T^{0}$ and a price $p$, the interim (conditional) expected utility of agent $i$ is defined as follows:

$$
U_{i}(z \mid p, q)=E\left\{u(i, z, t) \mid \tilde{p}=p, \tilde{t}_{i}=q\right\} .
$$

In the rational expectations equilibrium, an agent updates her belief on the distribution of signal profiles based on her own private signal and observation of the equilibrium price. She computes her expected utility with the updated belief and aims to maximize the interim expected utility subject to her budget constraint. The formal definition of the rational expectations equilibrium is given below.

Definition 1 (Rational Expectations Equilibrium (REE)) A rational expectations equilibrium for the private information economy $\mathcal{E}=\left\{I \times T, u, e, f,\left(\tilde{t}_{i}, i \in I\right), \tilde{s}\right\}$ is a pair of an allocation and a price process $\left(x^{*}, p^{*}\right)$ such that:

\footnotetext{
${ }^{8}$ The measure structure on the product space $I \times T$ will be specified in Section 6 .

${ }^{9} \mathrm{~A}$ vector $z$ is in $\mathbb{R}_{++}^{m}$ if and only if all of its components are positive.
} 
1. $x^{*}$ is feasible, i.e., $\int_{I} x^{*}\left(i, p^{*}(t), t_{i}\right) d \lambda=\int_{I} e\left(i, t_{i}\right) d \lambda$ for P-almost all $t \in T$;

2. for $\lambda$-almost all $i \in I$ and for $P$-almost all $t \in T, x^{*}\left(i, p^{*}(t), t_{i}\right)$ is a maximizer of the following problem:

$$
\begin{array}{ll}
\max & U_{i}\left(z \mid p^{*}(t), t_{i}\right) \\
\text { subject to } & z \in B_{i}\left(p^{*}(t), t_{i}\right) .
\end{array}
$$

The following is a notion of incentive compatibility in the setting of REE. It says that an agent cannot increase her interim expected utility by mis-reporting her private signal.

Definition 2 (Incentive Compatibility) $A R E E\left(x^{*}, p^{*}\right)$ is said to be incentive compatible if $\lambda$-almost all $i \in I$,

$$
U_{i}\left(x^{*}\left(i, p^{*}(t), t_{i}\right) \mid p^{*}(t), t_{i}\right) \geq U_{i}\left(x^{*}\left(i, p^{*}\left(t_{-i}, t_{i}^{\prime}\right), t_{i}\right) \mid p^{*}\left(t_{-i}, t_{i}^{\prime}\right), t_{i}\right)
$$

holds for all $t \in T$ and $t_{i}^{\prime} \in T^{0}$.

The following definitions of ex-post efficiency and interim efficiency are selfexplanatory.

Definition 3 (Ex-post Efficiency) A REE $\left(x^{*}, p^{*}\right)$ is said to be ex-post efficient if for $P$-almost all $t \in T$, there does not exist an integrable function $y_{t}$ from $I$ to $\mathbb{R}_{+}^{m}$ such that

1. $\int_{I} y_{t}(i) d \lambda=\int_{I} e\left(i, t_{i}\right) d \lambda$;

2. $u\left(i, y_{t}(i), t_{i}\right)>u\left(i, x^{*}\left(i, p^{*}(t), t_{i}\right), t\right)$ for $\lambda$-almost all $i \in I$.

Definition 4 (Interim Efficiency) $A R E E\left(x^{*}, p^{*}\right)$ is said to be interim efficient if for $P$-almost all $t \in T$, there does not exist an integrable function $y_{t}$ from $I$ to $\mathbb{R}_{+}^{m}$ such that

1. $\int_{I} y_{t}(i) d \lambda=\int_{I} e\left(i, t_{i}\right) d \lambda$;

2. $U_{i}\left(y_{t}(i) \mid p^{*}, t_{i}\right)>U_{i}\left(x^{*} \mid p^{*}, t_{i}\right)$ for $\lambda$-almost all $i \in I$.

\subsection{Rational expectations equilibrium with aggregate signals, incentive compatibility and efficiency}

In the standard REE model, agents use information transmitted by the equilibrium price to better estimate their utility. We shall now take a further step by allowing agents to use one more macroeconomic statistic, namely, the aggregate signals. We call such an equilibrium concept rational expectations equilibrium with aggregate signals. We follow the notations in Subsection 2.2. 
For any $t \in T$, the empirical signal distribution is $\lambda f_{t}^{-1}$, where $f_{t}=f(\cdot, t)$. For $q \in T^{0}, \lambda f_{t}^{-1}(\{q\})$ is the fraction of agents whose private signal is $q$ when the signal profile is $t \in T$. Let $\Delta\left(T^{0}\right)$ be the set of all probability distributions on $T^{0}$. It is clear that $\lambda f_{t}^{-1} \in \Delta\left(T^{0}\right)$. The empirical signal distribution process $\tilde{\mu}$ is a function from $T$ to $\Delta\left(T^{0}\right)$ such that $\tilde{\mu}(t)=\lambda f_{t}^{-1}$, which is also called the aggregate signals.

An agent can make contingent trades based on information transmitted by the equilibrium price as well as the private and aggregate signals. An allocation $x$ is a measurable mapping from $I \times \Delta_{m} \times T^{0} \times \Delta\left(T^{0}\right)$ to $\mathbb{R}_{+}^{m} \cdot{ }^{10}$ For $(i, p, q, \mu) \in I \times \Delta_{m} \times$ $T^{0} \times \Delta\left(T^{0}\right), x(i, p, q, \mu)$ is the consumption bundle of agent $i$ when the price is $p$, her private signal is $q$ and the empirical signal distribution is $\mu$.

Given a consumption bundle $z \in \mathbb{R}_{+}^{m}$, the interim (conditional) expected utility of agent $i$ is defined as follows.

$$
U_{i}(z \mid p, q, \mu)=E\left\{u(i, z, t) \mid \tilde{p}=p, \tilde{t}_{i}=q, \tilde{\mu}=\mu\right\}
$$

where $p \in \Delta_{m}$ is the price, $q \in T^{0}$ is the private signal of agent $i$ and $\mu \in \Delta\left(T^{0}\right)$ is the empirical signal distribution.

In the REE with aggregate signals, agent $i$ updates her belief on the distribution of signal profiles upon observing the price $p$, her own private signal $q$ and the empirical signal distribution $\mu$. Each agent aims to maximize her conditional expected utility subject to her budget constraint. We state the formal definition of REE with aggregate signals below.

Definition 5 (Rational Expectations Equilibrium (REE) with Aggregate Signals) Let $\mathcal{E}=\left\{I \times T, u, e, f,\left(\tilde{t}_{i}, i \in I\right), \tilde{s}\right\}$ be a private information economy. A REE with aggregate signals for $\mathcal{E}$ is a pair of an allocation and a price $\left(x^{*}, p^{*}\right)$ such that:

1. $x^{*}$ is feasible, i.e., $\int_{I} x^{*}\left(i, p^{*}(t), t_{i}, \lambda f_{t}^{-1}\right) d \lambda=\int_{I} e\left(i, t_{i}\right) d \lambda$ for P-almost all $t \in$ $T$, and

2. $x_{i}^{*}$ is a maximizer of the following problem:

$$
\begin{array}{ll}
\max & U_{i}\left(z \mid p^{*}(t), t_{i}, \lambda f_{t}^{-1}\right) \\
\text { s.t. } & z \in B_{i}\left(p^{*}(t), t_{i}\right)
\end{array}
$$

for $\lambda$-almost all $i \in I$ and $P$-almost all $t \in T$.

Condition (1) is standard. Condition (2) indicates that each agent, upon observing the price, and the private and aggregate signals, maximizes her interim expected utility subject to the budget constraint.

Since the aggregate signals depend on the "pooled" information, there is also the issue of incentive compatibility. Its formal definition is given below.

\footnotetext{
${ }^{10}$ The measurability is stated with respect to the usual product $\sigma$-algebra of $\mathcal{I}, \mathcal{B}_{\Delta_{m}}, \mathcal{T}^{0}$, and $\mathcal{B}_{\Delta\left(T^{0}\right)}$, where $\mathcal{B}_{\Delta_{m}}$ and $\mathcal{B}_{\Delta\left(T^{0}\right)}$ are the Borel algebras on $\Delta_{m}$ and $\Delta\left(T^{0}\right)$ respectively.
} 
Definition 6 (Incentive Compatibility) A REE with aggregate signals $\left(x^{*}, p^{*}\right)$ is said to be incentive compatible if $\lambda$-almost all $i \in I$,

$U_{i}\left(x_{i}^{*}\left(p^{*}(t), t_{i}, \lambda f_{t}^{-1}\right) \mid p^{*}(t), t_{i}, \lambda f_{t}^{-1}\right) \geq U_{i}\left(x_{i}^{*}\left(p^{*}\left(t_{-i}, t_{i}^{\prime}\right), t_{i}, \lambda f_{\left(t_{-i}, t_{i}^{\prime}\right)}^{-1}\right) \mid p^{*}\left(t_{-i}, t_{i}^{\prime}\right), t_{i}, \lambda f_{\left(t_{-i}, t_{i}^{\prime}\right)}^{-1}\right)$,

holds for all $t \in T$ and $t_{i}^{\prime} \in T^{0}$.

The following definition of interim efficiency is analogous to Definition 4.

Definition 7 (Interim Efficiency) A REE with aggregate signals $\left(x^{*}, p^{*}\right)$ is said to be interim efficient if for $P$-almost all $t \in T$, there does not exist an integrable function $y_{t}$ from $I$ to $\mathbb{R}_{+}^{m}$ such that

1. $\int_{I} y_{t}(i) d \lambda=\int_{I} e\left(i, t_{i}\right) d \lambda$;

2. $U_{i}\left(y_{t}(i) \mid p^{*}(t), t_{i}, \lambda f_{t}^{-1}\right)>U_{i}\left(x^{*} \mid p^{*}(t), t_{i}, \lambda f_{t}^{-1}\right)$ for $\lambda$-almost all $i \in I$.

\subsection{Some additional assumptions}

In order to study the relationship between the two REE notions introduced above and to prove our main result, we shall need to work with several assumptions on the private information economy. The first two assumptions are standard and self-explanatory. The latter two assumptions involve (conditional) independence of the private signal process $f$.

A1: For any fixed $i \in I$ and $t \in T$, the utility function $u(i, \cdot, t)$ is strictly concave.

A2: The utility function depends only on agent's private signal. That is, there is a function $v$ from $I \times \mathbb{R}_{+}^{m} \times T^{0}$ to $\mathbb{R}_{+}$such that for each $(i, x, t) \in I \times \mathbb{R}_{+}^{m} \times T$, $u(i, x, t)=v\left(i, x, t_{i}\right)$.

A3: The private signal process $f$ is essentially pairwise independent conditioned on the macro state of nature $\tilde{s}$. That is, for $\lambda$-almost all $i \in I, \tilde{t}_{i}$ and $\tilde{t}_{j}$ are independent conditioned on $\tilde{s}$ for $\lambda$-almost $j \in I$.

A4: The private signal process $f$ is essentially pairwise independent in the sense that for $\lambda$-almost all $i \in I, \tilde{t}_{i}$ and $\tilde{t}_{j}$ are independent for $\lambda$-almost all $j \in I{ }^{11}$

When assumption A3 or A4 is imposed, an immediate technical difficulty arises, which is the so-called measurability problem of independent processes. In our context, a signal process that is essentially independent, conditioned on the macro states of nature is never jointly measurable in the usual sense except for trivial cases. ${ }^{12}$ Hence,

\footnotetext{
${ }^{11}$ For a detailed discussion on pairwise (conditional) independence, see Sun (2006).

${ }^{12}$ See Proposition 2.1 in Sun (2006), and Proposition 4 in Hammond and Sun (2008) for detailed discussion of the measurability problem.
} 
we need to work with a joint agent-probability space $(I \times T, \mathcal{I} \otimes \mathcal{T}, \lambda \otimes P)$ that extends the usual measure-theoretic product $(I \times T, \mathcal{I} \otimes \mathcal{T}, \lambda \otimes P)$ of the agent space $(I, \mathcal{I}, \lambda)$ and the probability space $(T, \mathcal{T}, P)$, and retains the Fubini property. Below is a formal definition of the Fubini extension in Definition 2.2 of Sun (2006).

Definition 8 A probability space $(I \times \Omega, \mathcal{W}, Q)$ extending the usual product space $(I \times$ $\Omega, \mathcal{I} \otimes \mathcal{F}, \lambda \otimes P)$ is said to be a Fubini extension of $(I \times \Omega, \mathcal{I} \otimes \mathcal{F}, \lambda \otimes P)$ if for any real-valued $Q$-integrable function $f$ on $(I \times \Omega, \mathcal{W})$,

(1) the two functions $f_{i}$ and $f_{\omega}$ are integrable respectively on $(\Omega, \mathcal{F}, P)$ for $\lambda$ almost all $i \in I$, and on $(I, \mathcal{I}, \lambda)$ for $P$-almost all $\omega \in \Omega$;

(2) $\int_{\Omega} f_{i} d P$ and $\int_{I} f_{\omega} d P$ are integrable respectively on $(I, \mathcal{I}, \lambda)$ and $(\Omega, \mathcal{F}, P)$, with $\int_{I \times \Omega} f d Q=\int_{I}\left(\int_{\Omega} f_{i} d P\right) d \lambda=\int_{\Omega}\left(\int_{I} f_{\omega} d \lambda\right) d P .{ }^{13}$

To reflect the fact that the probability space $(I \times \Omega, \mathcal{W}, Q)$ has $(I, \mathcal{I}, \lambda)$ and $(\Omega, \mathcal{F}, P)$ as its marginal spaces, as required by the Fubini property, it will be denoted by $(I \times \Omega, \mathcal{I} \otimes \mathcal{F}, \lambda \otimes P)$.

Fix $s \in S$. For each $D \in \mathcal{I} \otimes \mathcal{T}$, let $\lambda \otimes P_{s}(D)=\lambda \otimes P\left(\left(I \times C_{s}\right) \cap D\right) / \pi_{s}$. Then, $\left(I \times T, \mathcal{I} \otimes \mathcal{T}, \lambda \otimes P_{s}\right)$ also extends the usual measure-theoretic product $(I \times$ $\left.T, \mathcal{I} \otimes \mathcal{T}, \lambda \otimes P_{s}\right)$, and retains the Fubini property. Thus, one can view $\lambda \otimes P_{s}$ as the conditional probability measure on $I \times T$, given $\tilde{s}=s$.

We shall assume that $f$ is a measurable process from $(I \times T, \mathcal{I} \otimes \mathcal{T})$ to $T^{0}$. For any $A \in \mathcal{T}$, the Fubini property associated with $(I \times T, \mathcal{I} \otimes \mathcal{T}, \lambda \otimes P)$ implies that $P\left(A \cap f_{i}^{-1}(\{q\})\right)$ is measurable in $i \in I$. This means that the process $f$ has event-wise measurable conditional probabilities, as defined in Definition 4 of Hammond and Sun (2008). By Theorem 1 of Hammond and Sun (2008), one can always find a real-valued random variable $\tilde{s}$ on $(T, \mathcal{T}, P)$ such that $f$ is essentially pairwise independent conditioned on $\tilde{s}$. The only restriction in this paper is to assume that $\tilde{s}$ takes finitely many values. Since the $\sigma$-algebra generated by a real-valued random variable is countably generated, and a countably generated $\sigma$-algebra can be approximated by $\sigma$-algebras generated by finite partitions in terms of information as shown in Allen (1983), the assumption that the private signals are essentially pairwise independent conditioned on finitely many macro states of nature (as in A3) is a reasonable assumption.

When the macro state is $s$, the signal distribution of agent $i$ conditioned on the macro state is $P_{s} f_{i}^{-1}$, i.e., the probability for agent $i$ to have $q \in T^{0}$ as her private signal is $P_{s}\left(f_{i}^{-1}(\{q\})\right)$, where $f_{i}=f(i, \cdot)$. Let $\mu_{s}$ be the agents' average signal distribution conditioned on the macro state $s$, i.e.,

$$
\mu_{s}(\{q\})=\int_{I} P_{s}\left(f_{i}^{-1}(\{q\})\right) d \lambda=\int_{I} \int_{T} 1_{\{q\}}(f(i, t)) d P_{s} d \lambda
$$

\footnotetext{
${ }^{13}$ The classical Fubini Theorem is only stated for the usual product measure spaces. It does not apply to integrable functions on $(I \times \Omega, \mathcal{W}, Q)$ since these functions may not be $\mathcal{I} \otimes \mathcal{F}$-measurable. However, the conclusions of that theorem do hold for processes on the enriched product space $(I \times$ $\Omega, \mathcal{W}, Q)$ that extends the usual product.
} 
where $1_{\{q\}}$ is the indicator function of the singleton set $\{q\}$. Throughout the rest of this paper, the following non-triviality assumption on the process $f$ will be imposed:

$$
\forall s, s^{\prime} \in S, s \neq s^{\prime} \Rightarrow \mu_{s} \neq \mu_{s^{\prime}} .
$$

This says that different macro states of nature correspond to different average conditional distributions of agents' signals.

\subsection{The relationship between the REE with aggregate signals and the REE}

One may ask naturally what the relationship between the REE and REE with aggregate signals is. When the REE price in either Definition 1 or Definition 5 fully reveals the macro states, ${ }^{14}$ the two notions are equivalent in the sense that one can be converted to the other.

Lemma 1 Assume A3. Let $\left(x^{*}, p^{*}\right)$ be a REE with aggregate signals. If $p^{*}$ fully reveals the macro states, then one can find an allocation $\bar{x}^{*}$ so that $\left(\bar{x}^{*}, p^{*}\right)$ is a REE. The other direction is also true.

Without the full revelation of the macro states, the two REE notions need not be equivalent in general. However, under the additional assumptions A1 and A2, the REE with aggregate signals and the standard REE are again equivalent when the equilibrium price only depends on the macro states (i.e., the equilibrium price partially reveals the macro states).

Lemma 2 Assume A1, A2 and A3. Then, for a REE with aggregate signals $\left(x^{*}, p^{*}\right)$, if $p^{*}$ only depends on the macro states, then one can find an allocation $\bar{x}^{*}$ so that $\left(\bar{x}^{*}, p^{*}\right)$ is a REE. The other direction is also true.

The proofs for Lemmas 1 and 2 are given in Subsections 6.2 and 6.3 respectively.

\section{The Main Result and its Corollaries}

If the private signals are essentially pairwise independent conditioned on the macro state of nature, ${ }^{15}$ then there is a REE with aggregate signals whose price depends only on the macro state of nature. In this case, each individual's private signal has negligible influence on the REE price and also on the aggregate signals, and therefore the REE is incentive compatible. This is the content of the following theorem.

\footnotetext{
${ }^{14}$ This means that the price depends only on the macro states, and different macro states correspond to different prices for those states up to null events. In other words, the price and $\tilde{s}$ generate essentially the same $\sigma$-algebras on $T$.

${ }^{15}$ For the existence of non-trivial independent processes that are measurable in a Fubini extension, see Section 5 of Sun (2006). It is shown in Sun and Zhang (2009) that one can take the relevant agent space to be an extension of the classical Lebesgue unit interval. For a most general existence result, see Podczeck (2010).
} 
Theorem 1 Under assumption $\mathbf{A 3}$, there exists an incentive compatible, interim effcient REE with aggregate signals in which the equilibrium price $p$ depends only on the macro state of nature.

When the economy has only idiosyncratic level information, i.e., the macro state variable $\tilde{s}$ is constant, then the conditional independence in assumption $\mathbf{A} 3$ becomes unconditional independence. This means that $\mathbf{A} 4$ is satisfied. In this case, Theorem 1 implies that the REE price with aggregate signals is constant and trivially reveals the macro states. Hence, as shown in the following corollary, Lemma 1 implies the existence of a REE in the usual sense.

Corollary 1 Under assumption $\mathbf{A} 4$, there exists an incentive compatible and interim efficient REE in which the equilibrium price is constant.

Suppose that the agents' utility functions are strictly concave and depend only on their noisy private signals. Lemma 2 then implies the equivalence of REE with aggregate signals and the standard REE. The existence of a standard REE follows as a corollary from Theorem 1 .

Corollary 2 Under assumptions $\mathbf{A} 1, \mathbf{A} 2$ and $\mathbf{A} 3$, there exists an incentive compatible and interim efficient REE in which the equilibrium price $p$ depends on the macro state of nature. Moreover, such a REE is ex-post efficient.

\section{Discussion}

In view of the non-existence example of Kreps (1977), the earlier contributions on the REE were focused on the generic existence (Radner (1979), Allen (1981)). By now, it is well-known that with a finite number of agents, the REE not only does not exist universally but also fails to be Pareto efficient and incentive compatible; see Glycopantis et al. (2005), p. 31 and also Example 9.1.1, p. 43.

In contrast to the above, the current paper demonstrates that the REE exists universally, and also is Pareto efficient and incentive compatible. Thus, the paper resolves the difficulties that the REE faces as a solution concept. Our results enable us to conclude that in the presence of a continuum of agents with non-trivial but negligible private information, the REE becomes an appealing concept as it does have desirable properties, contrary to the finite agent case.

There are several other papers dealing with the REE with a continuum of agents that we discuss below. We first discuss the relationship of our Corollaries 1 and 2 with related results in the literature. Our Theorem 1 proves some results about a new concept introduced in this paper, which have no analogs in the literature.

The early contributions by Einy et al. (2000a), Einy et. al. (2000b) consider an asymmetric information economy with a continuum of agents and a finite number of 
states of nature. The private information of each agent is a partition of the finite state space. Since a finite state space has only finitely many different partitions, we can find a partition on the measure space of agents so that all the agents in each partitioning set of agents share the same private information. Therefore, there is little heterogeneity on the private information side of their model. In contrast, our model allows agents to have non-trivial idiosyncratic private information. Hence, their work is not directly related to ours.

Another interesting paper by Heifetz and Minelli (2002) models the idea of informational smallness without aggregate uncertainty for an asymmetric information economy with a continuum of independent replica economies. The definition of an allocation in their paper is based on the notion of Pettis integral (Definition 6, p. 213). Instead of the usual state-wise feasibility condition, the authors used the condition that the value of the aggregate consumption is equal to the value of the aggregate endowment for every price function (see p. 212 and p. 213). Note that this does not imply market clearing, which requires the equality of aggregate consumption and aggregate endowment for almost all the state of nature. It seems to us that the reason they used this approach is to avoid the so-called measurability problem associated with a continuum of independent random variables. ${ }^{16}$ Our Corollary 1 has a similar flavor of a constant REE price as in their Theorem 2 on a continuum of independent replica economies. The main differences here are (1) we work with a general continuum economy rather than a replica economy; (2) we work with a general independence condition, and a Fubini extension, where the state-wise feasibility condition is fulfilled (see part 1 of Definition 1). Our Corollary 2 allows for a more general set-up where aggregate uncertainty is included in addition to idiosyncratic private information.

McLean and Postlewaite (2002, 2003, 2005) model the idea of informational smallness (i.e., roughly speaking, approximate perfect competition) in countable replica economies. Their main objective is to show the consistency of ex-post efficiency, ex ante core convergence and incentive compatibility. ${ }^{17}$ They do not consider the REE notion in those papers.

In Theorem 1, a new REE concept has been introduced, where each agent conditions her expected utility on the information generated by the equilibrium price, her private signal and the aggregate signals. Theorem 1 shows the existence of an incentive compatible, interim efficient REE with aggregate signals under the rather weak assumption that there are finitely many macro states of nature. This extension of the Radner-Allen REE model is different from previous work in the literature since none of these models take into account the information carried in the aggregate signals.

\footnotetext{
${ }^{16}$ See, for example, Sun (2006).

${ }^{17}$ The corresponding limiting results for a continuum of agents are considered in Sun and Yannelis (2007) and Sun and Yannelis (2008).
} 


\section{Concluding Remarks}

We model the REE notion in an asymmetric information economy where each agent's private signal has negligible influence on the equilibrium price. This way, we are able to overcome the problems of the universal existence, ex-post efficiency and incentive compatibility of the REE.

We also introduce a new notion of REE with aggregate signals, i.e., agents maximize their expected utility conditioned on their own private information, and the information generated by the equilibrium price and the aggregate signals. For such a REE notion we proved that it exists, is interim efficient and incentive compatible.

To the best of our knowledge, this is the first paper which models the REE notion with a continuum of agents where both aggregate uncertainty and idiosyncratic private information are included. In such a general model, the difficulties that one encounters in the finite agent setting are no longer present.

Finally, it should be noted that the exact limiting results in this paper on an atomless economy with asymmetric information have asymptotic analogs for large but finite asymmetric information economies. For the general methodology of obtaining asymptotic results, see Section 6 of Sun and Yannelis (2007).

\section{The Proofs}

\subsection{Proof of Theorem 1}

We outline the proof first. In step 1, we construct a large deterministic economy for each macro state of nature $s \in S$. Applying the standard Walrasian equilibrium existence results for a large deterministic economy, we can obtain a Walrasian equilibrium $\left(y_{s}, p_{s}\right)$ for such an economy. In step 2, we construct a price $p^{*}$ and an allocation $x^{*}$ from the collection of allocation-price pairs $\left\{\left(y_{s}, p_{s}\right): s \in S\right\}$, and show that $\left(x^{*}, p^{*}\right)$ is a REE with aggregate signals in which the equilibrium price depends only on the macro state of nature.

Step 1: Define a function $\Gamma$ from $I \times T$ to $I \times T^{0}$ by letting $\Gamma(i, t)=\left(i, t_{i}\right)$ for $(i, t) \in I \times T$. $\Gamma$ is a measurable mapping in the sense that $\Gamma^{-1}(D) \in \mathcal{I} \otimes \mathcal{T}$ for all $D \in \mathcal{I} \otimes \mathcal{T}^{0}$.

For each $s \in S$, let $\nu_{s}$ be the measure on $I \times T^{0}$ defined as

$$
\nu_{s}(D)=\left(\lambda \otimes P_{s}\right)\left(\Gamma^{-1}(D)\right)
$$

for $D \in \mathcal{I} \otimes \mathcal{T}^{0}$. For $(i, q) \in I \times T^{0}$, define a utility function $V_{s}(i, \cdot, q)$ on $\mathbb{R}_{+}^{m}$ by letting

$$
V_{s}(i, z, q)=E\left\{u(i, z, t) \mid \tilde{s}=s, \tilde{t}_{i}=q\right\}
$$

for $z \in \mathbb{R}_{+}^{m}$.

For each $s \in S$, we define a large deterministic economy $\overline{\mathcal{E}}_{s}=\left\{\left(I \times T^{0}, \mathcal{I} \otimes\right.\right.$ $\left.\left.\mathcal{T}^{0}, \nu_{s}\right), V_{s}, e\right\}$, where the utility function for agent $(i, q) \in I \times T^{0}$ is $V_{s}(i, \cdot, q)$, and the 
initial endowment for agent $(i, q)$ is $e(i, q)$. By the standard Walrasian equilibrium existence results (see, for example, Aumann (1966)), there is a Walrasian equilibrium allocation $y_{s}$ and a strictly positive equilibrium price $p_{s} \in \Delta_{m}$ for the economy $\overline{\mathcal{E}}_{s}$ such that:

1. $y_{s}$ is feasible, i.e., $\int_{I \times T^{0}} y_{s}(i, q) d \nu_{s}=\int_{I \times T^{0}} e(i, q) d \nu_{s}$, and

2. for $\nu_{s}$-almost all agent $(i, q) \in I \times T^{0}, y_{s}(i, q)$ is a maximal element in her budget set $B_{(i, q)}\left(p_{s}\right)=\left\{y \in \mathbb{R}_{+}^{m}: p_{s} y \leq p_{s} e(i, q)\right\}{ }^{18}$

Step 2: Define a mapping $x^{*}$ from $I \times \Delta_{m} \times T^{0} \times \Delta\left(T^{0}\right)$ to $\mathbb{R}_{+}^{m}$ by letting

$$
x^{*}(i, p, q, \mu)= \begin{cases}y_{s}(i, q) & \text { if } p=p_{s} \text { and } \mu=\mu_{s} \text { for some } s \in S \\ e(i, q) & \text { otherwise. }\end{cases}
$$

for each $(i, q) \in I \times T^{0}$.

By the non-triviality assumption in equation (4) in Subsection 2.4, there is at most one $s$ such that $\mu=\mu_{s}$. Hence, $x^{*}$ is well-defined.

Define the following sets

$$
\forall s \in S, L_{s}=\left\{t \in T: \lambda f_{t}^{-1}=\mu_{s}\right\} ; L_{0}=T-\cup_{s \in S} L_{s} .
$$

The non-triviality assumption in equation (4) implies that for any $s, s^{\prime} \in S$ with $s \neq s^{\prime}, L_{s} \cap L_{s^{\prime}}=\emptyset$. The measurability of the sets $L_{s}, s \in S$ and $L_{0}$ follows from the measurability of $f$. Thus, the collection $\left\{L_{0}\right\} \cup\left\{L_{s}, s \in S\right\}$ forms a measurable partition of $T$.

By equation (3) and the Fubini property for $\left(I \times T, \mathcal{I} \otimes \mathcal{T}, \lambda \otimes P_{s}\right)$, we have $\mu_{s}(\{q\})=\int_{I \times T} 1_{\{q\}}(f(i, t)) d\left(\lambda \otimes P_{s}\right)$ for any $q \in T^{0}$. Thus, $\mu_{s}$ is actually the distribution $\left(\lambda \otimes P_{s}\right) f^{-1}$ of $f$, viewed as a random variable on the product space $I \times T$. Since the signal process $f$ satisfies the condition of essential pairwise conditional independence, for $\lambda$-almost all $i \in I$, the random variables $f_{i}$ and $f_{j}$ from $\left(T, \mathcal{T}, P_{s}\right)$ to $X$ are independent for $\lambda$-almost all $j \in I$. The exact law of large numbers in Corollary 2.9 of Sun (2006) implies that $P_{s}\left(L_{s}\right)=1$ for each $s \in S$.

Choose a strictly positive price $p_{0} \in \Delta_{m}$ which is different from each $p_{s}, s \in S$. For each $t \in T$, define

$$
p^{*}(t)= \begin{cases}p_{s} & \text { if } t \in L_{s} \text { for some } s \in S \\ p_{0} & \text { otherwise }\end{cases}
$$

For any $s, s^{\prime} \in S, P_{s}\left(C_{s^{\prime}}\right)=P_{s}\left(L_{s^{\prime}}\right)$ is one when $s=s^{\prime}$ and zero when $s \neq s^{\prime}$. It is clear that $P\left(C_{s} \Delta L_{s}\right)=0$. Hence $p^{*}(t)$ depends essentially on the macro state of nature. For this reason, we can also write the price as $p(\tilde{s})$.

\footnotetext{
18 By modifying the values of $y_{s}$ on a null set (if necessary), we can assume that for every $(i, q) \in$ $I \times T^{0}, y_{s}(i, q)$ is a maximal element in her budget set.
} 
Since the society's signal distribution cannot be influenced by a single agent, we have for each $i \in I, \lambda f_{\left(t_{-i}, t_{i}\right)}^{-1}=\lambda f_{\left(t_{-i}, t_{i}^{\prime}\right)}^{-1}$ for any $t \in T$ and $t_{i}^{\prime} \in T^{0}$. This means that for any $i \in I, t \in T, t_{i}^{\prime} \in T^{0}$, and $s \in S$,

$$
t \in L_{s} \Leftrightarrow \lambda f_{t}^{-1}=\mu_{s} \Leftrightarrow \lambda f_{\left(t_{-i}, t_{i}^{\prime}\right)}^{-1}=\mu_{s} \Leftrightarrow\left(t_{-i}, t_{i}^{\prime}\right) \in L_{s} .
$$

Since $L_{0}$ is $T \backslash \cup_{s \in S} L_{s}$, we also know that $t \in L_{0} \Leftrightarrow\left(t_{-i}, t_{i}^{\prime}\right) \in L_{0}$. Hence, we have $p^{*}(t)=p^{*}\left(t_{-i}, t_{i}^{\prime}\right)$ for any $t \in T$ and $t_{i}^{\prime} \in T^{0}$. Therefore,

$$
\begin{aligned}
& U_{i}\left(x_{i}^{*}\left(p^{*}(t), t_{i}, \lambda f_{t}^{-1}\right) \mid p^{*}(t), t_{i}, \lambda f_{t}^{-1}\right)= \\
& U_{i}\left(x_{i}^{*}\left(p^{*}\left(t_{-i}, t_{i}^{\prime}\right), t_{i}, \lambda f_{\left(t_{-i}, t_{i}^{\prime}\right)}^{-1}\right) \mid p^{*}\left(t_{-i}, t_{i}^{\prime}\right), t_{i}, \lambda f_{\left(t_{-i}, t_{i}^{\prime}\right)}^{-1}\right),
\end{aligned}
$$

holds for all $t \in T$ and $t_{i}^{\prime} \in T^{0}$, i.e., the condition of incentive compatibility in Definition 6 is satisfied.

We will complete the proof by showing that $\left(x^{*}, p^{*}\right)$ is a REE with aggregate signals for the private information economy $\mathcal{E}$. By the definition of $\nu_{s}$, we have

$$
\int_{I \times T^{0}} y_{s}(i, q) d \nu_{s}=\int_{I \times T} y_{s}\left(i, t_{i}\right) d \lambda \otimes P_{s}
$$

and

$$
\int_{I \times T^{0}} e(i, q) d \nu_{s}=\int_{I \times T} e\left(i, t_{i}\right) d \lambda \otimes P_{s} .
$$

Given a macro state $s \in S$, assumption A3 implies the essential pairwise independence of the random variables $y_{s}\left(i, \tilde{t}_{i}\right) i \in I$ and the random variables $e\left(i, \tilde{t}_{i}\right) i \in I$ respectively. By the exact law of large numbers in Corollary 2.10 of Sun (2006), there exists $T_{s} \in \mathcal{T}$ with $P_{s}\left(T_{s}\right)=1$ such that for every $t \in T_{s}$,

$$
\int_{I} y_{s}\left(i, t_{i}\right) d \lambda=\int_{I \times T} y_{s}\left(i, t_{i}\right) d \lambda \otimes P_{s}
$$

and

$$
\int_{I} e\left(i, t_{i}\right) d \lambda=\int_{I \times T} e\left(i, t_{i}\right) d \lambda \otimes P_{s} .
$$

By equations (7) - (10), we have for any $t \in T_{s}$,

$$
\int_{I} y_{s}\left(i, t_{i}\right) d \lambda=\int_{I \times T^{0}} y_{s}(i, q) d \nu_{s}
$$

and

$$
\int_{I} e\left(i, t_{i}\right) d \lambda=\int_{I \times T^{0}} e(i, q) d \nu_{s}
$$

Since $y_{s}$ is a feasible allocation for the economy $\overline{\mathcal{E}}_{s}$, combined with equations (11) and (12), we have, for any $t \in T_{s}$,

$$
\int_{I} y_{s}\left(i, t_{i}\right) d \lambda=\int_{I} e\left(i, t_{i}\right) d \lambda .
$$


Let $\Omega_{0}=\bigcup_{s \in S} L_{s} \cap T_{s}$. Then $P\left(\Omega_{0}\right)=1$. For any $t \in \Omega_{0}$, there is a unique $s \in S$ such that $t \in L_{s} \cap T_{s}$, and thus $x^{*}\left(i, p^{*}(t), t_{i}, \lambda f_{t}^{-1}\right)=y_{s}\left(i, t_{i}\right)$ and furthermore

$$
\int_{I} x^{*}\left(i, p^{*}(t), t_{i}, \lambda f_{t}^{-1}\right) d \lambda=\int_{I} y_{s}\left(i, t_{i}\right) d \lambda=\int_{I} e\left(i, t_{i}\right) d \lambda
$$

Consequently, $x^{*}$ is feasible.

Note that for each $s \in S, \tilde{s}(t)=s, \tilde{\mu}(t)=\mu_{s}$, and $\tilde{p}^{*}(t)=p_{s}$ hold for $P$ almost all $t \in L_{s}$. Thus, the $\sigma$-algebras generated by the random variables $\tilde{s}$ and $\tilde{\mu}$ are essentially the same while the $\sigma$-algebra generated by $\tilde{p}^{*}$ is a sub- $\sigma$-algebra. Hence, for any $z \in \mathbb{R}_{+}^{m}$,

$$
\begin{aligned}
U_{i}\left(z \mid p_{s}, q, \mu_{s}\right) & =E\left\{u(i, z, t) \mid \tilde{p}^{*}=p_{s}, \tilde{t}_{i}=q, \tilde{\mu}=\mu_{s}\right\} \\
& =E\left\{u(i, z, t) \mid \tilde{s}=s, \tilde{t_{i}}=q\right\}=V_{s}(i, z, q) .
\end{aligned}
$$

It is left to show that for each agent $i \in I, x_{i}^{*}$ maximizes the conditional expected utility $U_{i}$ subject to her budget constraint.

As noted above, for any $t \in \Omega_{0}$, there is a unique $s \in S$ such that $t \in L_{s} \cap T_{s}$. The budget set for agent $i$ is $B_{i}\left(p^{*}(t), t_{i}\right)=B_{i}\left(p_{s}, t_{i}\right)=\left\{z \in \mathbb{R}_{+}^{m}: p_{s} z \leq p_{s} e\left(i, t_{i}\right)\right\}$. This is exactly the same as the budget set $B_{\left(i, t_{i}\right)}\left(p_{s}\right)$ of agent $\left(i, t_{i}\right)$ in the deterministic economy $\overline{\mathcal{E}}_{s}$. Since $V_{s}\left(i, \cdot, t_{i}\right)=U_{i}\left(\cdot \mid p^{*}(t), t_{i}, \lambda f_{t}^{-1}\right)$, the following two problems are equivalent:

Problem (I)

$$
\begin{array}{ll}
\max & V_{s}\left(i, z, t_{i}\right) \\
\text { subject to } & z \in B_{\left(i, t_{i}\right)}\left(p_{s}\right)
\end{array}
$$

and

$$
\text { Problem (II) }
$$

$$
\begin{array}{ll}
\max & U_{i}\left(z \mid p^{*}(t), t_{i}\right) \\
\text { subject to } & z \in B_{i}\left(p^{*}(t), t_{i}, \lambda f_{t}^{-1}\right)
\end{array}
$$

Since $y_{s}\left(i, t_{i}\right)$ is a maximizer for Problem (I), it must be a maximizer for Problem (II) as well. By definition, $x^{*}\left(i, p^{*}(t), t_{i}, \lambda f_{t}^{-1}\right)=y_{s}\left(i, t_{i}\right)$. Hence, $x^{*}\left(i, p^{*}(t), t_{i}, \lambda f_{t}^{-1}\right)$ is a maximizer for Problem (II). That is to say that $x^{*}\left(i, p^{*}(t), t_{i}, \lambda f_{t}^{-1}\right)$ maximizes $U_{i}\left(\cdot \mid p^{*}(t), t_{i}, \lambda f_{t}^{-1}\right)$ subject to the budget set $B_{i}\left(p^{*}(t), t_{i}\right)$.

Hence, $\left(x^{*}, p^{*}\right)$ constitutes a REE with aggregate signals for the private information economy $\mathcal{E}$.

Finally, we shall prove that $\left(x^{*}, p^{*}\right)$ is interim efficient. Suppose the two conditions in the definition of REE with aggregate signals hold for any $t \in \bar{T}$, where $\bar{T} \in \mathcal{T}$ and $P(\bar{T})=1$. Fix $t \in \bar{T}$, we can construct a large deterministic economy $\mathcal{E}_{t}=\left\{(I, \mathcal{I}, \lambda), \bar{u}_{t}, \bar{e}_{t}\right\}$, where $\bar{u}_{t}(i, \cdot)=U_{i}\left(\cdot \mid p^{*}(t), t_{i}, \lambda f_{t}^{-1}\right)$ and $\bar{e}_{t}(i)=e\left(i, t_{i}\right)$ for each agent $i \in I$. Let $\bar{p}_{t}=p^{*}(t)$ and $\bar{x}_{t}(i)=x^{*}\left(i, p^{*}(t), t_{i}, \lambda f_{t}^{-1}\right)$ for each $i \in I$. Then, it is easy to see that $\left(\bar{x}_{t}, \bar{p}_{t}\right)$ is a Walrasian equilibrium for $\mathcal{E}_{t}$. It follows that $\bar{x}_{t}$ is efficient in the sense that there is no allocation $\bar{y}$ for the economy $\mathcal{E}_{t}$ such that

$$
\int_{I} \bar{y}(i) d \lambda=\int_{I} \bar{e}_{t}(i) d \lambda
$$




$$
\bar{u}_{t}(i, \bar{y}(i))>\bar{u}_{t}\left(i, \bar{x}_{t}(i)\right) \text { for } \lambda \text {-almost all } i \in I .
$$

The above two conditions can be re-written as

$$
\begin{gathered}
\int_{I} \bar{y}(i) d \lambda=\int_{I} e\left(i, t_{i}\right) d \lambda \\
U_{i}\left(\bar{y} \mid p^{*}(t), t_{i}, \lambda f_{t}^{-1}\right)>U_{i}\left(x^{*}\left(i, p^{*}(t), t_{i}, \lambda f_{t}^{-1}\right) \mid p^{*}(t), t_{i}, \lambda f_{t}^{-1}\right) \text { for } \lambda \text {-almost all } i \in I .
\end{gathered}
$$

Hence, $\left(x^{*}, p^{*}\right)$ is interim efficient. Q.E.D.

\subsection{Proof of Lemma 1}

An equilibrium price $p^{*}(t)$ is said to fully reveal the macro states if the price depends only on the macro states, and different macro states correspond to different prices for those states. That means $p^{*}(t)$ is constant $p_{s}^{*}$ on $C_{s}$, and $p_{s}^{*} \neq p_{s^{\prime}}^{*}$ when $s \neq s^{\prime}$. In the following proof, we assume $p^{*}(t)$ fully reveals the macro states.

We first prove one part of the equivalence result by converting a REE with aggregate signals $\left(x^{*}, p^{*}\right)$ to a standard REE. We can define an allocation $\bar{x}^{*}$ for the REE notion by letting

$$
\bar{x}^{*}(i, p, q)= \begin{cases}x^{*}\left(i, p, q, \mu_{s}\right) & \text { if } p=p_{s}^{*} \text { for some } s \in S \\ e(i, q) & \text { otherwise. }\end{cases}
$$

By the feasibility of $x^{*}$ (see Definition 5), we have

$$
\int_{I} x^{*}\left(i, p^{*}(t), t_{i}, \lambda f_{t}^{-1}\right) d \lambda=\int_{I} e\left(i, t_{i}\right) d \lambda
$$

for $P$-almost all $t \in T$. As in the proof of Theorem 1, A3 and the exact law of large numbers in Corollary 2.9 of Sun (2006) imply that for $P$-almost all $t \in C_{s}, \lambda f_{t}^{-1}=\mu_{s}$. Thus, by the full revelation property of $p^{*}$, we also know that for $P$-almost all $t \in T$, there exists a unique $s \in S$ such that $p^{*}(t)=p_{s}^{*}$ and $\lambda f_{t}^{-1}=\mu_{s}$. Hence, we obtain that $\bar{x}^{*}\left(i, p^{*}(t), t_{i}\right)=x^{*}\left(i, p^{*}(t), t_{i}, \lambda f_{t}^{-1}\right)$ for $P$-almost all $t \in T$. This implies the feasibility of $\bar{x}^{*}$ in REE (see Definition 1)

$$
\int_{I} \bar{x}^{*}\left(i, p^{*}(t), t_{i}\right) d \lambda=\int_{I} e\left(i, t_{i}\right) d \lambda
$$

for $P$-almost all $t \in T$.

Since both the REE price $p^{*}$ and the aggregate signals $\lambda f_{t}^{-1}$ fully reveal the macro states, then $\tilde{s}, p^{*}$ and $\lambda f_{t}^{-1}$ generate the same partition modulo the null events. Hence, for $P$-almost all $t \in T$,

$$
U_{i}\left(\cdot \mid p^{*}(t), t_{i}, \lambda f_{t}^{-1}\right)=U_{i}\left(\cdot \mid p^{*}(t), t_{i}\right) .
$$


Therefore, it follows from condition (2) of Definition 5 that for $\lambda$-almost all $i \in I$, and for $P$-almost all $t \in T, \bar{x}^{*}\left(i, p^{*}(t), t_{i}\right)$ is a maximizer of the following problem:

$$
\begin{array}{ll}
\max & U_{i}\left(z \mid p^{*}(t), t_{i}\right) \\
\text { subject to } & z \in B_{i}\left(p^{*}(t), t_{i}\right) .
\end{array}
$$

Hence, $\left(\bar{x}^{*}, p^{*}\right)$ is a REE as in Definition 1.

For the other direction of the equivalence result, fix a standard REE $\left(x^{*}, p^{*}\right)$. We can define an allocation $\bar{x}^{*}$ for the REE with aggregate signals as follows:

$$
\bar{x}^{*}(i, p, q, \mu)= \begin{cases}x^{*}(i, p, q) & \text { if } p=p_{s}^{*} \text { and } \mu=\mu_{s} \text { for some } s \in S \\ e(i, q) & \text { otherwise. }\end{cases}
$$

As above, we obtain that for $P$-almost all $t \in T, \bar{x}^{*}\left(i, p^{*}(t), t_{i}, \lambda f_{t}^{-1}\right)=x^{*}\left(i, p^{*}(t), t_{i}\right)$, and

$$
\int_{I} \bar{x}^{*}\left(i, p^{*}(t), t_{i}, \lambda f_{t}^{-1}\right) d \lambda=\int_{I} x^{*}\left(i, p^{*}(t), t_{i}\right) d \lambda=\int_{I} e\left(i, t_{i}\right) d \lambda .
$$

Hence, $\bar{x}^{*}$ is feasible.

As above, equation (18) still holds. It follows from condition (2) of Definition 1 that for $\lambda$-almost all $i \in I$, and for $P$-almost all $t \in T, \bar{x}^{*}\left(i, p^{*}(t), t_{i}, \lambda f_{t}^{-1}\right)$ is a maximizer of the following problem:

$$
\begin{array}{ll}
\max & U_{i}\left(z \mid p^{*}, t_{i}, \lambda f_{t}^{-1}\right) \\
\text { s.t. } & z \in B_{i}\left(p^{*}(t), t_{i}\right)
\end{array}
$$

Hence, $\left(\bar{x}^{*}, p^{*}\right)$ is a REE with aggregate signals as in Definition 5. Q.E.D.

\subsection{Proof of Lemma 2}

When $p^{*}(t)$ depends only on the macro states, we have $p^{*}(t)=p_{s}^{*}$ for $t \in C_{s}$. In the following proof, we assume $p^{*}$ possesses this property.

Since assumption A2 holds, there is a function $v$ from $I \times \mathbb{R}_{+}^{m} \times T^{0}$ to $\mathbb{R}_{+}$such that for each $(i, x, t) \in I \times \mathbb{R}_{+}^{m} \times T, u(i, x, t)=v\left(i, x, t_{i}\right)$. Thus, we can work with the utility function $v$. It follows from equations (1) and (2) that

$$
U_{i}(\cdot \mid p, q, \mu)=U_{i}(\cdot \mid p, q)=v(i, \cdot, q)
$$

As in the proof of Lemma 1, we first convert a REE with aggregate signals $\left(x^{*}, p^{*}\right)$ to a standard REE. We define

$$
\bar{x}^{*}(i, p, q)= \begin{cases}x^{*}\left(i, p, q, \mu_{s}\right) & \text { if } p=p_{s}^{*} \text { for some } s \in S \\ e(i, q) & \text { otherwise. }\end{cases}
$$

As in the proof of Lemma 1 , when $p^{*}$ fully reveals the macro states, the choice of $s$ is unique in the above definition. Without full revelation property of $p^{*}$, there may exist 
two distinct states $s$ and $s^{\prime}$ such that $p_{s}^{*}=p_{s^{\prime}}^{*}$. This causes ambiguity in the above definition. However, we shall justify below that such ambiguity is irrelevant under assumptions A1, A2 and A3.

It follows from equation (19) that given a private signal $q \in T^{0}$ and price $p$, agent $i$ faces the following maximization problem in the definition of REE with aggregate signals:

$$
\begin{array}{ll}
\max & v_{i}(z, q) \\
\text { s.t. } & z \in B_{i}(p, q) .
\end{array}
$$

Since $u_{i}$ (and hence $v_{i}$ ) is strictly concave by assumption $\mathbf{A} \mathbf{1}$, the solution for the above problem is unique. It follows immediately that $x^{*}\left(i, p_{s}^{*}, q, \mu_{s}\right)=x^{*}\left(i, p_{s^{\prime}}^{*}, q, \mu_{s^{\prime}}\right)$ when $p_{s}^{*}=p_{s^{\prime}}^{*}$ in that they are the solution of the same problem. Hence, the choice of $s$ is irrelevant in the definition of $\bar{x}^{*} . \bar{x}^{*}$ is thus well-defined. This also implies that for $P$-almost all $t \in T, \bar{x}^{*}\left(i, p^{*}(t), t_{i}\right)=x^{*}\left(i, p^{*}(t), t_{i}, \lambda f_{t}^{-1}\right)$, which in turn implies the feasibility of $\bar{x}^{*}$ in REE as $x^{*}$ is feasible in REE with aggregate signals.

It follows from equation (19) and condition (2) of Definition 5 that for $\lambda$-almost all $i \in I$, and for $P$-almost all $t \in T, \bar{x}^{*}\left(i, p^{*}(t), t_{i}\right)$ is a maximizer of the following problem:

$$
\begin{array}{ll}
\max & U_{i}\left(z \mid p^{*}(t), t_{i}\right) \\
\text { subject to } & z \in B_{i}\left(p^{*}(t), t_{i}\right) .
\end{array}
$$

Hence, $\left(\bar{x}^{*}, p^{*}\right)$ is a REE as in Definition 1.

The other direction of the equivalence result can be readily obtained by constructing a similar allocation as in the second part of the proof for Lemma 1. Q.E.D.

\subsection{Proof of Corollary 1}

By Theorem 1 and assumption A4, there exists an interim efficient REE with aggregate signals $\left(x^{*}, p^{*}\right)$ in which the equilibrium price $p^{*}$ is constant. As in the proof of Lemma 1 , we can construct a standard REE $\left(\bar{x}^{*}, p^{*}\right)$. This ensures the existence of a standard REE with constant equilibrium price under assumption A4.

Since $p^{*}$ is constant, $p^{*}\left(t_{-i}, t_{i}\right)=p^{*}\left(t_{-i}, t_{i}^{\prime}\right)$ for all $t \in T$ and $t_{i}^{\prime} \in T^{0}$. This indicates that $\left(\bar{x}^{*}, p^{*}\right)$ satisfies the incentive compatibility condition in Definition 2 since

$$
U_{i}\left(x^{*}\left(i, p^{*}(t), t_{i}\right) \mid p^{*}(t), t_{i}\right)=U_{i}\left(x^{*}\left(i, p^{*}\left(t_{-i}, t_{i}^{\prime}\right), t_{i}\right) \mid p^{*}\left(t_{-i}, t_{i}^{\prime}\right) .\right.
$$

It is left to show that $\left(\bar{x}^{*}, p^{*}\right)$ is interim efficient. We prove it by contradiction. If $\left(\bar{x}^{*}, p^{*}\right)$ is not interim efficient, then there exists $A \in \mathcal{I}$ with $P(A)>0$ such that for all $t \in A$, there exists an integrable function $y_{t}$ from $I$ to $\mathbb{R}_{+}^{m}$ with the following properties

1. $\int_{I} y_{t}(i) d \lambda=\int_{I} e\left(i, t_{i}\right) d \lambda$

2. $U_{i}\left(y_{t}(i) \mid p^{*}, t_{i}\right)>U_{i}\left(\bar{x}^{*} \mid p^{*}, t_{i}\right)$ for $\lambda$-almost all $i \in I$. 
Since $\bar{x}^{*}\left(i, p^{*}(t), t_{i}\right)=x^{*}\left(i, p^{*}(t), t_{i}, \lambda f_{t}^{-1}\right)$ and $U_{i}\left(\cdot \mid p^{*}(t), t_{i}, \lambda f_{t}^{-1}\right)=U_{i}\left(\cdot \mid p^{*}(t), t_{i}\right)$ for $P$-almost all $t \in T$ (see the proof of Lemma 1), the second property of $y_{t}$ above implies

$$
U_{i}\left(y_{t}(i) \mid p^{*}, t_{i}, \lambda f_{t}^{-1}\right)>U_{i}\left(x^{*} \mid p^{*}, t_{i}, \lambda f_{t}^{-1}\right) \text { for } \lambda-\text { almost all } i \in I .
$$

This, combined with the first property, contradicts the interim efficiency condition of $\left(x^{*}, p^{*}\right)$ for the REE with aggregate signals (see Definition 7$)$. We can thus conclude that $\left(\bar{x}^{*}, p^{*}\right)$ is interim efficient. Q.E.D.

\subsection{Proof of Corollary 2}

Under assumptions A1, A2 and A3, Theorem 1 holds. We follow the proof of Theorem 1 to obtain a REE with aggregate signals $\left(x^{*}, p^{*}\right)$. Then, following the proof of Lemma 2 , we obtain a standard REE $\left(\bar{x}^{*}, p^{*}\right)$ in which the equilibrium price depends only on the macro states. Since $p^{*}\left(t_{-i}, t_{i}\right)=p^{*}\left(t_{-i}, t_{i}^{\prime}\right)$ for all $t \in T$ and $t_{i}^{\prime} \in T^{0},\left(\bar{x}^{*}, p^{*}\right)$ satisfies the incentive compatibility condition in Definition 2. Interim efficiency can be proved in the same way as in the proof for Corollary 1. By equation (19), interim efficiency and ex-post efficiency are equivalent (see Definitions 3 and 4$)$. Thus $\left(\bar{x}^{*}, p^{*}\right)$ is also ex-post efficient. Q.E.D. 


\section{References}

B. Allen, Generic existence of completely revealing equilibria with uncertainty, when prices convey information, Econometrica 49 (1981), 1173-1199.

B. Allen, Neighboring information and distributions of agents characteristics under uncertainty, Journal of Mathematical Economics 12 (1983), 63-101.

R. J. Aumann, Existence of competitive equilibria in markets with a continuum of traders, Econometrica 34 (1966), 1-17.

E. Einy and D. Moreno and B. Shitovitz, Rational expectations equilibria and the ex-post core of an economy with asymmetric information, Journal of Mathematical Economics 34 (2000), 527-535.

E. Einy and D. Moreno and B. Shitovitz, On the core of an economy with differential information, Journal of Economic Theory 94 (2000), 262-270.

D. Glycopantis, A. Muir and N.C. Yannelis, Non-implementation of rational expectations as a perfect Bayesian equilibrium, Economic Theory 26 (2005), 765-791.

D. Glycopantis and N.C. Yannelis, Differential Information Economies, Studies in Economic Theory, 19, Springer, 2005.

P. J. Hammond and Y. N. Sun, Monte Carlo simulation of macroeconomic risk with a continuum of agents: the general case, Economic Theory 36 (2008), 303-325.

A. Heifetz and E. Minelli, Informational smallness in rational expectations equilibria, Journal of Mathematical Economics 38 (2002), 197-218.

M. D. Kreps, A note on fulfilled expectations equilibria, Journal of Economic Theory 14 (1977), 32-43.

R. McLean and A. Postlewaite, Informational size and incentive compatibility, Econometrica 70 (2002), 2421-2453.

R. McLean and A. Postlewaite, Informational size and incentive compatibility with aggregate uncertainty, Games and Economic Behavior 45 (2003), 410-433.

R. McLean and A. Postlewaite, Core convergence with asymmetric information, Games and Economic Bahavior 50 (2005), 58-78.

K. Podczeck, On existence of rich Fubini extentions, Economic Theory 45 (2010), 1-22.

R. Radner, Rational expectation equilibrium: generic existence and information revealed by prices, Econometrica 47 (1979), 655-678.

Y. N. Sun, The exact law of large numbers via Fubini extension and characterization of insurable risks, Journal of Economic Theory 126 (2006), 31-69. 
Y. N. Sun and N. C. Yannelis, Perfect competition in asymmetric information economies: compatibility of efficiency and incentives, Journal of Economic Theory 134 (2007) 175-194.

Y. N. Sun and N.C. Yannelis, Ex ante efficiency implies incentive compatibility, Economic Theory 36 (2008), 35-55.

Y.N. Sun and Y.C. Zhang, Individual risk and Lebesgue extension without aggregate uncertainty, Journal of Economic Theory 144 (2009), 432-443. 